\title{
Exponential Synchronization of Stochastic Complex Dynamical Networks with Impulsive Perturbations and Markovian Switching
}

\author{
Wuneng Zhou, Anding Dai, Dongbing Tong, and Jun Yang \\ College of Information Science and Technology, Donghua University, Shanghai 201620, China \\ Correspondence should be addressed to Wuneng Zhou; zhouwuneng@163.com and Anding Dai; daianding41@163.com
}

Received 27 November 2013; Accepted 2 January 2014; Published 24 February 2014

Academic Editor: Zhengguang Wu

Copyright (c) 2014 Wuneng Zhou et al. This is an open access article distributed under the Creative Commons Attribution License, which permits unrestricted use, distribution, and reproduction in any medium, provided the original work is properly cited.

This paper investigates the exponential synchronization problem of stochastic complex dynamical networks with impulsive perturbation and Markovian switching. The complex dynamical networks consist of $\kappa$ modes, and the networks switch from one mode to another according to a Markovian chain with known transition probability. Based on the Lyapunov function method and stochastic analysis, by employing $M$-matrix approach, some sufficient conditions are presented to ensure the exponential synchronization of stochastic complex dynamical networks with impulsive perturbation and Markovian switching, and the upper bound of impulsive gain is evaluated. At the end of this paper, two numerical examples are included to show the effectiveness of our results.

\section{Introduction}

Since Watts and Strogatz wrote their pioneering work [1], complex dynamical networks have received a lot of research attention. One of the most important research topics in complex dynamical networks is synchronization which is studied as a common phenomenon of a population of dynamical interacting units $[2,3]$. Moreover, many different regimes of synchronization have been investigated, such as complete synchronization, phase synchronization, exponential synchronization, cluster synchronization, lag synchronization, and generalized synchronization [4-12].

Exponential synchronization is a more favorite property since it gives a fast convergence rate to the synchronous solution. In [7], exponential synchronization strategy for complex dynamical networks is proposed by using sampleddata control. Mean square exponential synchronization in Lagrange sense for uncertain complex dynamical networks is proposed in [12]. In [13], the adaptive synchronization issue of stochastic delayed neural networks with Markovian switching is considered and several sufficient conditions to ensure adaptive exponential synchronization in $p$ th moment of stochastic delayed neural networks with Markovian switching are derived.

It has been widely reported that networks have finite modes which switch from one mode to another at different times, and such a switching signal can be governed by a Markovian chain. Markovian jump networks are of great significance in modeling a class of complex networks with finite network modes, and many relevant results have been reported in the literature (see, e.g., [13-17], and the references therein). In [18], it has been revealed that a class of neural networks has finite modes that switch from one to another according to a Markovian chain with known transition probability. In [19], the exponential synchronization problem for an array of $N$ linearly coupled complex networks with Markovian switching and mixed time-delays is investigated. In [20], a sensor network has been shown to have jumping behavior due to the network's working environment and the mobility of sensor node. In [21], the exponential stability problem of stochastic neural networks with both Markovian jump parameters and mixed time delays is investigated and some sufficient conditions are derived by linear matrix inequality approach. In [22], the problem of sampled-data 
synchronization for Markovian jump neural networks with time-varying delay and variable samplings is considered, where the model-independent controller has been proposed via the LMI technique. In [23], the passivity analysis has been conducted for discrete-time stochastic neural networks with both Markovian jumping parameters and mixed time delays, and a delay-dependent passivity condition is derived by introducing a Lyapunov functional that accounts for the mixed time delays.

In the real world, there exist a number of networks in which the state of nodes is usually subject to instantaneous perturbations and experiences abrupt change at certain instants which may be caused by switching phenomena, frequent change, and other sudden noise. Such networks are described by impulsive differential networks [24, 25]. Stability and synchronization problems of impulsive differential networks have sparked the interest of many researchers. In [26], the authors' concern with the issues of synchronization of complex delayed dynamical networks with impulsive effects and several criteria to ensure the exponential synchronization of the complex delayed dynamical networks are established. The average consensus in delayed networks of dynamic agents with impulsive effects is investigated [27]. What is more, impulsive control as a special impulsive effect with the impulsive gain $b_{k} \in(-2,0)$ has been widely applied to ecosystems, financial systems, mechanical systems with impacts, and orbital transfer of satellite. In [28], the robust impulsive synchronization of coupled delayed neural networks with uncertainties is considered, and several criteria to ensure the robust synchronization of coupled networks are obtained by employing impulsive controller. Two different types of pinning impulsive control strategy are proposed in $[29,30]$. These literatures all consider a special case: the network impulses are generated simultaneously, which means that all nodes engender impulse at some certain time while in reality; there may be only some nodes that engender impulse at some certain time. In addition, the impulse generation may be provided by the network model switching and noise perturbations, but few literatures focus on network model switching, noise perturbations, and impulse perturbations together. If a network model contains random switching, noise, and impulse perturbations, how to realize exponential synchronization for the class of complex networks is very interesting. What is more, it is very important for us to understand exponential synchronization dynamical evolution for this class of networks.

In this paper, we are concerned with the analysis issue for exponential synchronization of stochastic complex dynamical networks with impulsive perturbations and Markovian switching. According to two different cases of complex dynamical networks, synchronous networks and asynchronous networks, some sufficient conditions are presented to ensure the exponential synchronization of stochastic complex dynamical network with impulsive perturbations and Markovian switching, and the upper bound of impulsive gain is evaluated. Two numerical examples are included to show the effectiveness of our results. The main contributions of this paper can be highlighted as follows.
(1) Impulsive effects, noise perturbations, and switchings are considered for modeling the coupled complex networks simultaneously, which has been rarely investigated.

(2) By using the average dwell time approach, M-matrix approach, Lyapunov theory, and stochastic analysis, some sufficient conditions are presented to ensure the exponential synchronization of stochastic complex dynamical networks with impulsive perturbations and Markovian switching.

The notations are quite standard. Throughout this paper, $R_{+}, R^{n}$, and $R^{n \times n}$ denote the set of nonnegative real numbers, $n$ dimensional Euclidean space, and the set of all $n \times n$ real matrices, respectively. The superscript $T$ denotes matrix transposition, trace $(\cdot)$ denotes the trace of the corresponding matrix, and $I$ denotes the identity matrix. $|\cdot|$ stands for the Euclidean norm in $R^{n}$. $\operatorname{diag}\{\cdots\}$ stands for the block diagonal matrix, and $\lambda_{\max }(A)$ denote the largest eigenvalue of symmetric matrix $A$.

\section{Model and Preliminaries}

Let $\{r(t), t \geq 0\}$ be a right continuous Markovian chain in a complete probability space $(\Omega, \mathscr{F}, P)$ taking values in a finite state set $S=\{1,2, \ldots, \kappa\}$ with generator $\Pi=\left(\pi_{i j}\right)_{\kappa \times \kappa}$ given by

$$
P\{r(t+\delta)=j \mid r(t)=i\}= \begin{cases}\pi_{i j} \delta+o(\delta), & i \neq j \\ 1+\pi_{i i} \delta+o(\delta), & i=j\end{cases}
$$

where $\delta>0$ and $\pi_{i j} \geq 0$ are the transition rate from $i$ to $j$ if $i \neq j$, while $\pi_{i i}=-\sum_{j \neq i} \pi_{i j}$.

In this paper, we consider a class of stochastic complex dynamical networks, which is described as follows:

$$
\begin{aligned}
& d x_{i}(t) \\
& =\left[\tilde{f}\left(x_{i}(t), r(t)\right)+c(r(t)) \sum_{j=1}^{N} a_{i j}(r(t)) \Gamma(r(t)) x_{j}(t)\right] d t \\
& \quad+\widetilde{g}\left(t, x_{i}(t), r(t)\right) d \omega(t), \quad i=1,2, \ldots, N,
\end{aligned}
$$

where $\{r(t), t>0\}$ is the continuous time Markov process, $x_{i}(t)=\left[x_{i 1}(t), x_{i 2}(t), \ldots, x_{i n}(t)\right]^{T} \in R^{n}$ is the state vector of node $i$ at time $t, \tilde{f}\left(x_{i}(t), r(t)\right)=$ $\left[\tilde{f}_{1}\left(x_{i 1}(t), r(t)\right), \tilde{f}_{2}\left(x_{i 2}(t), r(t)\right), \ldots, \tilde{f}_{n}\left(x_{i n}(t), r(t)\right)\right]^{T}$ is continuous vector value functions, the noise perturbation $\widetilde{g}(\cdot, \cdot, \cdot): R \times R^{n} \times S \rightarrow R^{n \times m}$ is a Borel measurable matrix function, $\omega(t) \in R^{m}$ is an $m$-dimensional Brownian motion, $\Gamma(r(t))=\operatorname{diag}\left(\gamma_{1, r(t)}, \gamma_{2, r(t)}, \ldots, \gamma_{n, r(t)}\right)$ is an inner coupling matrix between two connected nodes, $c(r(t))$ is the coupling strength, and $A(r(t))=\left(a_{i j}(r(t))\right) \in R^{N \times N}$ is coupling matrix which represents the topological structure of the whole network, where the entries $a_{i j}(r(t))$ are defined as follows: if nodes $i$ and $j(i \neq j)$ are connected, then 
$a_{i j}(r(t))=a_{j i}(r(t))>0$; otherwise $a_{i j}(r(t))=0$, and the diagonal entries of coupling matrix $A(r(t))$ are defined by

$$
a_{i i}(r(t))=-\sum_{j=1, j \neq i}^{N} a_{i j}(r(t))=-\sum_{j=1, j \neq i}^{N} a_{j i}(r(t)) .
$$

One important consideration in practical networks is the existence of impulsive perturbations and the impulse of each node which does not emerge at the same time. Hence, the impulsive perturbations network is described by

$$
\begin{aligned}
d x_{i}(t)= & {\left[\tilde{f}\left(x_{i}(t), r(t)\right)+c(r(t))\right.} \\
& \left.\quad \times \sum_{j=1}^{N} a_{i j}(r(t)) \Gamma(r(t)) x_{j}(t)+u_{i}(t)\right] d t \\
& +\tilde{g}\left(t, x_{i}(t), r(t)\right) d \omega(t),
\end{aligned}
$$

where

$$
u_{i}(t)=\sum_{k^{\prime}=1}^{\infty} b_{i k^{\prime}}\left(x_{i}\left(t_{k^{\prime}}^{-}\right)-s(t)\right) \delta\left(t-t_{k^{\prime}}^{i}\right)
$$

where $b_{i k^{\prime}}$ is the $i$ th node impulsive gain at $t=t_{k^{\prime}}^{i}, \delta(t)$ is the Dirac delta function, and $s(t)$ is a solution of an isolated node described by

$$
d s(t)=\tilde{f}(s(t), r(t)) d t+\widetilde{g}(t, s(t), r(t)) d \omega(t) .
$$

For each $i$, the discrete set $\left\{t_{k^{\prime}}^{i}\right\}$ satisfies $0 \leq t_{0^{\prime}}^{i}<t_{1^{\prime}}^{i}<\cdots<$ $t_{k^{\prime}}^{i}<\cdots, t_{k^{\prime}}^{i} \rightarrow+\infty$ as $k^{\prime} \rightarrow+\infty, x_{i}\left(t_{k^{\prime}}^{i-}\right)=\lim _{t \rightarrow t_{k^{\prime}}^{i-}} x(t)$, and $x_{i}\left(t_{k^{\prime}}^{i+}\right)=\lim _{t \rightarrow t_{k^{\prime}}^{i+}} x(t)=x_{i}\left(t_{k^{\prime}}^{i}\right)$.

For all $i$ and $k^{\prime}$, rearrange $\left\{t_{k^{\prime}}^{i}\right\}$, with sequence from small to large, and pick out the same elements, getting a new sequence $\left\{t_{k}\right\}$, such that $t_{0}<t_{1}<\cdots<t_{k}<\cdots$. Equivalently, the network (4) can be rewritten as

$$
\begin{aligned}
& \begin{array}{l}
d x_{i}(t) \\
=\left[\tilde{f}\left(x_{i}(t), r(t)\right)+c(r(t)) \sum_{j=1}^{N} a_{i j}(r(t)) \Gamma(r(t)) x_{j}(t)\right] d t \\
\quad+\tilde{g}\left(t, x_{i}(t), r(t)\right) d \omega(t), \quad t \neq t_{k} \\
\Delta x_{i}\left(t_{k}\right)=x_{i}\left(t_{k}^{+}\right)-x_{i}\left(t_{k}^{-}\right)=b_{i k}\left(x_{i}\left(t_{k}^{-}\right)-s(t)\right), \\
t=t_{k}, k \in Z^{+}, i=1,2, \ldots, N,
\end{array}
\end{aligned}
$$

where $b_{i k}=b_{i k^{\prime}}$, when $t_{k}=t_{k^{\prime}}^{i}$; otherwise, $b_{i k}=0$. For each $t_{k}$, there exists at least $t_{k^{\prime}}^{i}$ such that $t_{k^{\prime}}^{i}=t_{k}$.

Remark 1. In [24], the authors divide impulses into three forms: synchronizing impulses, desynchronizing impulses, and inactive impulses. When impulsive gain $b_{i k} \in(-2,0)$, these impulses belong to synchronizing impulses, which means that the impulses are beneficial to synchronization of impulsive dynamical network. When $b_{i k} \in(-\infty,-2) \cup(0,+\infty)$, these impulses attribute to desynchronizing impulses; that is, the impulsive effects can suppress the synchronization of the impulsive dynamical network. When $b_{i k}=-2$ or $b_{i k}=0$, these impulses pertain to inactive impulses, which is neither harmful nor beneficial to the synchronization of impulsive dynamical network.

Remark 2. In [25], authors consider the synchronization problem of coupled neural networks with Markovian switching and impulsive effects, in which the impulsive effects can occur not only at the instants coinciding with the system switching but also at the instants when there is no system switching. The model in this paper is similar to the one in [25], but it also has some differences when comparing both of them; that is, the model in this paper also considers the following situations which are not considered in [25]. First, impulses may not happen at the same time, that is, only a part of nodes possesses impulse at time $t_{k}$. Then, impulse may be synchronizing impulses, desynchronizing impulses, and inactive impulses.

The primary object here is to deal with the exponential synchronization problem of the stochastic complex dynamical network (7) and derive sufficient conditions such that the network (7) will synchronize into the desired trajectory $s(t)$.

Define $e_{i}(t)=x_{i}(t)-s(t)(i=1,2, \ldots, N)$ as the synchronization error; then the error system can be described by the following differential equations:

$$
\begin{gathered}
d e_{i}(t) \\
=\left[f\left(e_{i}(t), r(t)\right)+c(r(t)) \sum_{j=1}^{N} a_{i j}(r(t)) \Gamma(r(t)) e_{j}(t)\right] d t \\
+g\left(t, e_{i}(t), r(t)\right) d \omega(t), \quad t \neq t_{k} \\
\Delta e_{i}\left(t_{k}\right)=e_{i}\left(t_{k}^{+}\right)-e_{i}\left(t_{k}^{-}\right)=b_{i k} e_{i}\left(t_{k}^{-}\right), \\
t=t_{k}, \quad k \in Z^{+}, \quad i=1,2, \ldots, N,
\end{gathered}
$$

where $f\left(e_{i}(t), r(t)\right)=\tilde{f}\left(x_{i}(t), r(t)\right)-\tilde{f}(s(t), r(t))$ and $g(t, e(t), r(t))=\tilde{g}\left(t, x_{i}(t), r(t)\right)-\tilde{g}(t, s(t), r(t))$.

For the purpose of the exponential synchronization of the stochastic complex dynamical network (7), we need the following assumptions.

Assumption 3. The function $\tilde{f}(x(t), r(t))$ can be divided into two parts as follows:

$$
\tilde{f}(x(t), r(t))=C(r(t)) x(t)+\tilde{f}_{1}(x(t), r(t)),
$$

where $C(r(t)) \in R^{n \times n}$ is a constant matrix and $\widetilde{f}_{1}(x(t), r(t))$ is a nonlinear function satisfying the Lipschitz condition. That is, for any $i \in S$, there exists a constant $l_{i}>0$ such that

$$
\left|\tilde{f}_{1}(x, i)-\tilde{f}_{1}(y, i)\right| \leq l_{i}|x-y|, \quad \forall x, y \in R^{n} .
$$


Assumption 4. The noise intensity matrix $\tilde{g}(t, x(t), r(t))$ satisfies the bounded condition. That is, for any $i \in S$, there exists a constant $h_{i}>0$ such that

$$
\begin{aligned}
& \operatorname{trace}\left[(\widetilde{g}(t, x, i)-\widetilde{g}(t, y, i))^{T}(\tilde{g}(t, x, i)-\widetilde{g}(t, y, i))\right] \\
& \leq h_{i}|x-y|^{2}, \quad \forall x, y \in R^{n} .
\end{aligned}
$$

In order to derive the main results, the following definitions and lemmas are necessary in this paper.

Consider a stochastic differential equation with Markovian switching of the form

$$
d x(t)=f(t, r(t), x(t)) d t+g(t, r(t), x(t)) d \omega(t)
$$

on $t \in[0,+\infty)$ with the initial data given by $x(0) \in R^{n}$.

Definition 5 (see [31]). System (12) is said to be exponentially stable in mean square if there exist two constants $M_{0}>0$ and $\eta>0$ such that

$$
E\left\{|x(t)|^{2}\right\} \leq M_{0} e^{-\eta t}, \quad i=1,2, \ldots, N,
$$

for all initial conditions $x(0) \in R^{n}$.

Definition 6 (see [11]). The dynamical network (7) is said to be exponential synchronization in mean square if network (8) is exponentially stable in mean square.

Definition 7 (see [24], average impulsive interval). The average impulsive interval of the impulsive sequence $\zeta=$ $\left\{t_{1}, t_{2}, \ldots\right\}$ is equal to $T_{a}$ if there exists a positive integer $N_{0}$ and a positive number $T_{a}$, such that

$$
\frac{T-t}{T_{a}}-N_{0} \leq N_{\zeta}(T, t) \leq \frac{T-t}{T_{a}}+N_{0}, \quad \forall T \geq t \geq 0,
$$

where $N_{\zeta}(T, t)$ denotes the number of impulsive times of the impulsive sequence $\zeta$ on the interval $(t, T)$.

Lemma 8 (see [31], Dynkin formula). Let $V \in C^{2,1}\left(R_{+} \times\right.$ $\left.S \times R^{n}, R_{+}\right)$and $0 \leq \tau_{1} \leq \tau_{2}$ be bounded stopping times. If $V(t, i, x(t))$ and $\mathscr{L} V(t, i, x(t))$ are bounded on $t \in\left[\tau_{1}, \tau_{2}\right]$ with probability 1 , then

$$
\begin{aligned}
E V\left(\tau_{2}, r\left(\tau_{2}\right), x\left(\tau_{2}\right)\right)= & E V\left(\tau_{1}, r\left(\tau_{1}\right), x\left(\tau_{1}\right)\right) \\
& +E \int_{\tau_{1}}^{\tau_{2}} \mathscr{L} V(s, r(s), x(s)) d s .
\end{aligned}
$$

\section{Main Result}

In this section, we propose some criteria of exponential synchronization in mean square for stochastic complex dynamical networks with impulsive perturbations and Markovian switching.

Theorem 9. Let Assumptions 3 and 4 hold, and the average impulsive interval of the impulsive sequence $\zeta=\left\{t_{1}, t_{2}, \ldots\right\}$ is no less than $T_{a}$. Then, the coupled network (8) is globally exponentially stable in mean square with the convergence rate $\varepsilon$ if the following conditions are satisfied.
(1) $M=-\operatorname{diag}\left\{\eta_{1}, \eta_{2}, \ldots, \eta_{\kappa}\right\}-\Pi$ is a nonsingular $M$ matrix where $\eta_{i}=\lambda_{\max }\left\{C_{i}^{T}+C_{i}+\left(2 l_{i}+h_{i}\right) I_{n}\right\}$. In this case, there exists a positive constant $\alpha \gg 0$ such that $\left(q_{1}, q_{2}, \ldots, q_{\kappa}\right)^{T}=M^{-1} \vec{\alpha} \gg 0$, where $\vec{\alpha}=$ $(\alpha, \alpha, \ldots, \alpha)^{T}$.

(2) $\left|1+b_{i k}\right| \leq \sqrt{q / \widetilde{q}} e^{(1 / 2)(\alpha / \widetilde{q}-\varepsilon) T_{a}}$, where $q=\min \left\{q_{i}, i \in S\right\}$ and $\widetilde{q}=\max \left\{q_{i}, i \in S\right\}$.

Proof. Choose a nonnegative Lyapunov function as follows:

$$
V(t, r(t), e(t))=\frac{q_{r(t)}}{2} \sum_{i=1}^{N} e_{i}^{T}(t) e_{i}(t), \quad r(t) \in S .
$$

For $\left[t_{k}, t_{k+1}\right)$, note that almost every sample path $r(t)$ is a right-continuous step function with a finite number of simple jumps on $\left[t_{k}, t_{k+1}\right)$.

Without any loss of generality, we assume that there are $l$ jump points; that is, $t_{k}=t_{k, 0}<t_{k, 1}<\cdots<t_{k, l}<t_{k, l+1}=t_{k+1}$ (notice that $t_{k}$ and $t_{k+1}$ may not be jump points). It means that $r(t)$ takes unique values in $S$ when $t \in\left[t_{k, s}, t_{k, s+1}\right), s=$ $0,1, \ldots, l$.

Fix $s$ and assume that $r(t)=\sigma$; then the Lyapunov function can be rewritten as

$$
V(t, \sigma, e(t))=\frac{q_{\sigma}}{2} \sum_{i=1}^{N} e_{i}^{T}(t) e_{i}(t), \quad t \in\left[t_{k, s}, t_{k, s+1}\right)
$$

For each $\sigma$, computing $\mathscr{L} V(t, \sigma, e(t))$ along the trajectory of error system (8), one can obtain that

$$
\begin{aligned}
\mathscr{L} V(t, \sigma, e(t))= & q_{\sigma} \sum_{i=1}^{N} e_{i}^{T}(t)\left[f\left(e_{i}(t), \sigma\right)+c_{\sigma} \sum_{j=1}^{N} a_{i j}^{\sigma} \Gamma_{\sigma} e_{j}(t)\right] \\
& +\sum_{j=1}^{\kappa} \pi_{\sigma j} V(t, j, e(t)) \\
& +q_{\sigma} \sum_{i=1}^{N} \frac{1}{2} \operatorname{trace}\left[g^{T}\left(t, e_{i}(t), \sigma\right) g\left(t, e_{i}(t), \sigma\right)\right] \\
= & q_{\sigma} \sum_{i=1}^{N}\left[e_{i}^{T}(t)\left(C_{\sigma} e_{i}(t)+f_{1}\left(e_{i}(t), \sigma\right)\right)\right. \\
& +\frac{q_{\sigma}}{2} \sum_{i=1}^{N} \operatorname{trace}\left[g^{T}\left(t, e_{i}(t), \sigma\right) g\left(t, e_{i}(t), \sigma\right)\right] . \\
& \left.+c_{j=1}^{\kappa} \sum_{j=1}^{N} a_{i j}^{\sigma} e_{i}^{T}(t) \Gamma_{j} e_{j}(t)\right] e_{i}^{T}(t) e_{i}(t)
\end{aligned}
$$


According to Assumptions 3 and 4, we have

$$
\begin{aligned}
& \mathscr{L} V(t, \sigma, e(t)) \\
& \leq q_{\sigma} \sum_{i=1}^{N}\left[e_{i}^{T}(t)\left(\frac{1}{2}\left(C_{\sigma}^{T}+C_{\sigma}\right)+\left(l_{\sigma}+\frac{1}{2} h_{\sigma}\right) I_{n}\right) e_{i}(t)\right. \\
& \left.+c_{\sigma} \sum_{j=1}^{N} a_{i j}^{\sigma} e_{i}^{T}(t) \Gamma_{\sigma} e_{j}(t)\right] \\
& +\frac{1}{2} \sum_{j=1}^{\kappa} \pi_{\sigma j} q_{j} \sum_{i=1}^{N} e_{i}^{T}(t) e_{i}(t) \\
& \leq \sum_{i=1}^{N} \frac{1}{2}\left(q_{\sigma} \eta_{\sigma}+\sum_{j=1}^{\kappa} \pi_{\sigma j} q_{j}\right) e_{i}^{T}(t) e_{i}(t) \\
& +q_{\sigma} c_{\sigma} \sum_{i=1}^{N} \sum_{j=1}^{N} a_{i j}^{\sigma} e_{i}^{T}(t) \Gamma_{\sigma} e_{j}(t) \\
& =\sum_{j=1}^{n} \widetilde{e}_{j}^{T}(t)\left[\frac{1}{2}\left(\eta_{\sigma} q_{\sigma}+\sum_{k=1}^{\kappa} \pi_{\sigma k} q_{k}\right) I_{N}+q_{\sigma} c_{\sigma} \gamma_{j, \sigma} A_{\sigma}\right] \widetilde{e}_{j}(t),
\end{aligned}
$$

where $\widetilde{e}_{j}(t)=\left(e_{1 j}(t), e_{2 j}(t), \ldots, e_{N j}(t)\right)^{T}, f_{1}\left(e_{i}(t), \sigma\right)=$ $\tilde{f}_{1}\left(x_{i}(t), \sigma\right)-\tilde{f}_{1}(s(t), \sigma)$, and $\eta_{\sigma}=\lambda_{\max }\left(C_{\sigma}^{T}+C_{\sigma}+\left(2 l_{\sigma}+h_{\sigma}\right) I_{n}\right)$.

Consider that the coupling matrix $A_{\sigma}$ is symmetric; then it is not difficult to verify that

$$
\tilde{e}_{j}^{T}(t) A_{\sigma} \widetilde{e}_{j}(t) \leq \lambda_{\max }\left(A_{\sigma}\right) \tilde{e}_{j}^{T}(t) \tilde{e}_{j}(t)=0 .
$$

According to condition (2) of Theorem 9, we have

$$
\mathscr{L} V(t, \sigma, e(t)) \leq-\frac{\alpha}{2} \sum_{j=1}^{n} \tilde{e}_{j}^{T}(t) \widetilde{e}_{j}(t) \leq-p V(t, \sigma, e(t)),
$$

where $p=\alpha / \widetilde{q}$ and $\widetilde{q}=\max \left\{q_{i}, i \in S\right\}$.

Let $W(t, r(t), e(t))=e^{p t} V(t, r(t), e(t))$; then

$$
\begin{aligned}
& \mathscr{L} W(t, r(t), e(t)) \\
& \quad=p e^{p t} v(t, r(t), e(t))+e^{p t} \mathscr{L} V(t, r(t), e(t)) \\
& \quad \leq p e^{p t} v(t, r(t), e(t))-p e^{p t} v(t, r(t), e(t))=0 .
\end{aligned}
$$

Based on Lemma 8, we have

$$
\begin{aligned}
E W(t, r(t), e(t))= & E W\left(t_{k}, r\left(t_{k}\right), e\left(t_{k}\right)\right) \\
& +E \int_{t_{k}}^{t} \mathscr{L} W(s, r(s), e(s)) d s \\
\leq & E V\left(t_{k}, r\left(t_{k}\right), e\left(t_{k}\right)\right) .
\end{aligned}
$$

It is easy to get

$E V(t, r(t), e(t)) \leq E V\left(t_{k}, r\left(t_{k}\right), e\left(t_{k}\right)\right) e^{-p\left(t-t_{k}\right)}, \quad k \in Z^{+}$.
On the other hand, from the construction of $V(t, r(t), e(t))$, we have

$$
\begin{aligned}
V\left(t_{k}, r\left(t_{k}\right), e\left(t_{k}\right)\right) & =\frac{q_{r\left(t_{k}\right)}}{2} \sum_{i=1}^{N}\left(1+b_{i k}\right)^{2} e_{i}^{T}\left(t_{k}^{-}\right) e_{i}\left(t_{k}^{-}\right) \\
& =\frac{q_{r\left(t_{k}\right)}}{2 q_{r\left(t_{k}^{-}\right)}} q_{r\left(t_{k}^{-}\right)} \sum_{i=1}^{N}\left(1+b_{i k}\right)^{2} e_{i}^{T}\left(t_{k}^{-}\right) e_{i}\left(t_{k}^{-}\right) \\
& \leq \rho V\left(t_{k}^{-}\right), \quad \forall k \in Z^{+},
\end{aligned}
$$

where $\rho=\max \left\{(\tilde{q} / q)\left(1+b_{i k}\right)^{2}, i=1,2, \ldots, N, k \in Z^{+}\right\}$.

For $t \in\left[t_{k}, t_{k+1}\right), k \in Z^{+}$, combining (14) and condition (4) of Theorem 9 , we get

$$
\begin{aligned}
E V(t, r(s), e(t)) \leq & \rho E V\left(t_{k}^{-}, r\left(t_{k}^{-}\right), e\left(t_{k}^{-}\right)\right) e^{-p\left(t-t_{k}\right)} \\
& \leq \cdots \leq E V\left(t_{0}, r\left(t_{0}\right), e\left(t_{0}\right)\right) \rho^{k} e^{-p\left(t-t_{0}\right)} \\
& =E V\left(t_{0}, r\left(t_{0}\right), e\left(t_{0}\right)\right) e^{-p\left(t-t_{0}\right)+k \ln \rho} \\
& \leq E V\left(t_{0}, r\left(t_{0}\right), e\left(t_{0}\right)\right) \\
& \times e^{-\left(p-\ln \rho / T_{a}\right)\left(t-t_{0}\right)+N_{0} \ln \rho} \\
\leq & \widetilde{M} e^{-\varepsilon\left(t-t_{0}\right)},
\end{aligned}
$$

where $\widetilde{M}=E V\left(t_{0}, r\left(t_{0}\right), e\left(t_{0}\right)\right) e^{N_{0} \ln \rho}$.

It is easy to see that

$$
E e_{i}^{T}(t) e_{i}(t) \leq \frac{1}{\tilde{q}} E V(t, \sigma, e(t)) \leq \frac{\widetilde{M}}{\widetilde{q}} e^{-\varepsilon\left(t-t_{0}\right)} .
$$

The proof is completed.

Remark 10. We assume that there exist infinite time points $t_{k}$ at which both impulses and Markovian switches happen. In Theorem 9, we have derived a sufficient condition to guarantee Markovian switching and impulse interference network to achieve exponential mean-square synchronization, and we have evaluated the upper bound of impulsive gain. What is more, the proof of Theorem $9 \prod_{i=1}^{k}\left(q_{t_{i}} / q_{t_{i}^{-}}\right)$is magnified into $(q / \widetilde{q})^{k}$, which is not essential for $\Pi_{i=1}^{k}\left(q_{t_{i}} / q_{t_{i}^{-}}\right) \leq M_{0}$, where $M_{0}$ is a positive constant. For example, the time points are finite numbers when impulses and Markovian switches happen at the same time, so it is easy to get $\Pi_{i=1}^{k}\left(q_{t_{i}} / q_{t_{i}^{-}}\right) \leq$ $M_{0}$. It will engender impulse every time when network mode changes; thus, $\Pi_{i=1}^{k}\left(q_{t_{k}} / q_{t_{k}^{-}}\right)=q_{r\left(t_{k}\right)} / q_{r\left(t_{1}^{-}\right)} \leq M_{0}$. If $q_{t_{k}} \geq$ $q_{t_{k}^{-}}$, when impulse and switch happen at the same time, then $\Pi_{i=1}^{k}\left(q_{t_{k}} / q_{t_{k}^{-}}\right) \leq 1$. So, for $\Pi_{i=1}^{k}\left(q_{t_{i}} / q_{t_{i}^{-}}\right) \leq M_{0}$, the conditions of Theorem 9 can be weakened, which is shown in Corollary 11.

Corollary 11. Let Assumptions 3 and 4 be true, and also assume that for each $k \in Z^{+}, \Pi_{i=1}^{k}\left(q_{t_{i}} / q_{t_{i}^{-}}\right) \leq M_{0}$ holds. 
And the average impulsive interval of the impulsive sequence $\zeta=\left\{t_{1}, t_{2}, \ldots\right\}$ is no less than $T_{a}$. Then, the network (8) is globally exponentially stable in mean square with the convergence rate $\varepsilon$ if the following conditions are satisfied.

(1) $M=-\operatorname{diag}\left\{\eta_{1}, \eta_{2}, \ldots, \eta_{\kappa}\right\}-\Pi$ is a nonsingular $M-$ matrix where $\eta_{i}=\lambda_{\max }\left\{C_{i}^{T}+C_{i}+\left(2 l_{i}+h_{i}\right) I_{n}\right\}$. In this case, there exists a positive constant $\alpha \gg 0$ such that $\left(q_{1}, q_{2}, \ldots, q_{\kappa}\right)^{T}=M^{-1} \vec{\alpha} \gg 0$, where $\vec{\alpha}=$ $(\alpha, \alpha, \ldots, \alpha)^{T}$.

(2) $\left|1+b_{i k}\right| \leq e^{(1 / 2)(\alpha / \widetilde{q}-\varepsilon) T_{a}}$, where $q=\min \left\{q_{i}, i \in S\right\}$ and $\tilde{q}=\max \left\{q_{i}, i \in S\right\}$.

Remark 12. In Theorem 9, there is a rigorous requirement on networks that the condition $M=-\operatorname{diag}\left\{\eta_{1}, \eta_{2}, \ldots, \eta_{\kappa}\right\}-\Pi$ is a nonsingular $M$-matrix. There are some cases such that $M$ unsatisfies the above condition. For example, if $\eta_{i}>0$, for all $i \in S$, then $M$ is not a nonsingular $M$-matrix. In this case, the network (8) may be not stable even without impulse interference (desynchronizing impulses). In this condition, it will be interesting and significant to achieve network synchronization via the design of impulsive controller.

Assume that $b_{i k^{\prime}}=b_{k}$ and $t_{k^{\prime}}^{i}=t_{k}$ for all $i$, we can derive the synchronization criteria of the network (2) via impulsive control, which is given as follows.

Theorem 13. Let Assumptions 3 and 4 hold, and the average impulsive interval of the impulsive sequence $\zeta=\left\{t_{1}, t_{2}, \ldots\right\}$ is no more than $T_{a}$. Then, the network (8) via impulsive control is globally exponentially stable in mean square with the convergence rate $\varepsilon$ if the following conditions are satisfied.

(1) $M=\operatorname{diag}\left\{\eta_{1}, \eta_{2}, \ldots, \eta_{\kappa}\right\}+\Pi$ is a nonsingular $M-$ matrix where $\eta_{i}=\lambda_{\max }\left\{C_{i}^{T}+C_{i}+\left(2 l_{i}+h_{i}\right) I_{n}\right\}>0$. Hence, there exists a positive constant $\alpha \gg 0$ such that $\left(q_{1}, q_{2}, \ldots, q_{\kappa}\right)^{T}=M^{-1} \vec{\alpha} \gg 0$, where $\vec{\alpha}=$ $(\alpha, \alpha, \ldots, \alpha)^{T}$.

(2) $\left|1+b_{i k}\right| \leq \sqrt{q / \widetilde{q}} e^{-(1 / 2)(\alpha / q+\varepsilon) T_{a}}$, where $q=\min \left\{q_{i}, i \in S\right\}$ and $\tilde{q}=\max \left\{q_{i}, i \in S\right\}$.

Proof. The Lyapunov function is the same as that in Theorem 9. Computing $L V(t, \sigma, e(t))$ along the trajectory of error system (8), similar to the process in Theorem 9, we can get

$$
\begin{aligned}
& L V(t, \sigma, e(t)) \\
& \qquad q_{\sigma} \sum_{i=1}^{N} e_{i}^{T}(t)\left[\frac{1}{2}\left(C_{\sigma}^{T}+C_{\sigma}\right)+\left(l_{\sigma}+\frac{1}{2} h_{\sigma}\right) I_{n}\right] e_{i}(t) \\
& \quad+q_{\sigma} c_{\sigma} \sum_{i=1}^{N} \sum_{j=1}^{N} a_{i j}^{\sigma} e_{i}^{T}(t) \Gamma_{\sigma} e_{j}(t)
\end{aligned}
$$

$$
\begin{aligned}
& +\sum_{j=1}^{\kappa} \pi_{\sigma j} q_{j} \sum_{i=1}^{N} e_{i}^{T}(t) e_{i}(t) \\
\leq & \sum_{j=1}^{n} \widetilde{e}_{j}^{T}(t)\left[\frac{1}{2}\left(\eta_{\sigma} q_{\sigma}+\sum_{k=1}^{\kappa} \pi_{\sigma k} q_{k}\right) I_{N}+q_{\sigma} c_{\sigma} \gamma_{j, \sigma} A_{\sigma}\right] \widetilde{e}_{j}(t) \\
\leq & \frac{\alpha}{2} \sum_{j=1}^{N} \widetilde{e}_{j}^{T}(t) \widetilde{e}_{j}(t)=\frac{\alpha}{2} \sum_{i=1}^{N} e_{i}^{T}(t) e_{i}(t)
\end{aligned}
$$

where $\eta_{\sigma}=\lambda_{\max }\left\{C_{\sigma}^{T}+C_{\sigma}+\left(2 l_{\sigma}+h_{\sigma}\right) I_{n}\right\}$.

Based on Lemma 8, we have

$$
\begin{aligned}
E V(t, r(t), e(t))= & E V\left(t_{k}, r\left(t_{k}\right), e\left(t_{k}\right)\right) \\
& +E \int_{t_{k}}^{t} \mathscr{L} V(s, r(s), e(s)) d s \\
\leq & E V\left(t_{k}, r\left(t_{k}\right), e\left(t_{k}\right)\right) \\
& +\int_{t_{k}}^{t} \frac{\alpha}{2} E \sum_{i=1}^{N} e_{i}^{T}(s) e_{i}(s) d s .
\end{aligned}
$$

Because of $(q / 2) \sum_{i=1}^{N} e_{i}^{T}(t) e_{i}(t) \leq V(t, r(t), e(t))$, we have

$$
\begin{aligned}
E\left(\sum_{i=1}^{N} e_{i}^{T}(t) e_{i}(t)\right) \leq & \frac{2}{q} E V\left(t_{k}, r\left(t_{k}\right), e\left(t_{k}\right)\right) \\
& +\int_{t_{k}}^{t} \frac{\alpha}{q} E \sum_{i=1}^{N} e_{i}^{T}(s) e_{i}(s) d s .
\end{aligned}
$$

It follows from the Gronwall's inequality that

$$
E\left(\sum_{i=1}^{N} e_{i}^{T}(t) e_{i}(t)\right) \leq \frac{2}{q} E V\left(t_{k}, r\left(t_{k}\right), e\left(t_{k}\right)\right) e^{(\alpha / q)\left(t-t_{k}\right)}
$$

$\forall k \in Z^{+}$

On the other hand, from the construction of $V(t, r(t), e(t))$, we have

$$
\begin{aligned}
V\left(t_{k}, r\left(t_{k}\right), e\left(t_{k}\right)\right) & =\frac{q_{r\left(t_{k}\right)}}{2} \sum_{i=1}^{N}\left(1+b_{i k}\right)^{2} e_{i}^{T}\left(t_{k}^{-}\right) e_{i}\left(t_{k}^{-}\right) \\
& =\frac{q_{r\left(t_{k}\right)}}{2 q_{r\left(t_{k}^{-}\right)}} q_{r\left(t_{k}^{-}\right)} \sum_{i=1}^{N}\left(1+b_{i k}\right)^{2} e_{i}^{T}\left(t_{k}^{-}\right) e_{i}\left(t_{k}^{-}\right) \\
& \leq \rho V\left(t_{k}^{-}\right), \quad \forall k \in Z^{+},
\end{aligned}
$$

where $\rho=\max \left\{(\tilde{q} / q)\left(1+b_{i k}\right)^{2}, i=1,2, \ldots, N, k \in Z^{+}\right\}$.

The proof can be completed by following the same steps as that in Theorem 9.

For each $k \in Z^{+}$, if $\Pi_{i=1}^{k}\left(q_{t_{i}} / q_{t_{i}^{-}}\right) \leq M_{0}$ holds, we have the following corollary. 
Corollary 14. Let Assumptions 3 and 4 be true, and the average impulsive interval of the impulsive sequence $\zeta=$ $\left\{t_{1}, t_{2}, \ldots\right\}$ is no more than $T_{a}$. Then, the network (8) via impulsive control is globally exponentially stable in mean square with the convergence rate $\varepsilon$ if the following conditions are satisfied.

(1) $M=\operatorname{diag}\left\{\eta_{1}, \eta_{2}, \ldots, \eta_{\kappa}\right\}+\Pi$ is a nonsingular $M$ matrix where $\eta_{i}=\lambda_{\max }\left\{C_{i}^{T}+C_{i}+\left(2 l_{i}+h_{i}\right) I_{n}\right\}$. Hence, there exists a positive constant $\alpha \gg 0$ such that $\left(q_{1}, q_{2}, \ldots, q_{\kappa}\right)^{T}=M^{-1} \vec{\alpha} \gg 0$, where $\vec{\alpha}=$ $(\alpha, \alpha, \ldots, \alpha)^{T}$.

(2) $\left|1+b_{i k}\right| \leq e^{-(1 / 2)((\alpha / q)+\varepsilon) T_{a}}$, where $q=\min \left\{q_{i}, i \in S\right\}$ and $\widetilde{q}=\max \left\{q_{i}, i \in S\right\}$.

\section{Numerical Simulation}

In this section, we present two numerical simulations to illustrate the feasibility and effectiveness of our results.

4.1. Example 1. Consider that a stochastic complex network model consists of five nodes and two modes, which is described as follows:

$$
\begin{aligned}
d x_{i}(t)= & {\left[C(r(t)) x_{i}(t)+B(r(t)) f\left(x_{i}(t)\right)+c(r(t))\right.} \\
& \left.\times \sum_{j=1}^{5} a_{i j}(r(t)) \Gamma(r(t)) x_{j}(t)\right] d t \\
& +g\left(t, x_{i}(t), r(t)\right) d \omega(t), \quad t \neq t_{k}
\end{aligned}
$$

where $r(t)$ is a Markov chain in the state space $S=\{1,2\}$ with the generator $\Pi_{1}=\left[\begin{array}{cc}-10 & 10 \\ 2 & -2\end{array}\right], x_{i}(t)=\left[x_{i 1}(t), x_{i 2}(t)\right]^{T}$, $f(x)=\tanh (x), c_{1}=2, c_{2}=1, \Gamma_{1}=I_{2}, \Gamma_{2}=0.8 I_{2}, C_{1}=$ $\left[\begin{array}{cc}-3.1 & 0.5 \\ 0.5 & -3.8\end{array}\right], C_{2}=\left[\begin{array}{cc}-3.5 & 0.5 \\ 0.5 & -3.6\end{array}\right], B_{1}=\left[\begin{array}{cc}1.2 & -0.6 \\ -0.6 & -1.2\end{array}\right], B_{2}=\left[\begin{array}{cc}1.1 & -0.8 \\ -0.8 & 1.2\end{array}\right]$, $g_{1}\left(x_{i}(t)\right)=0.5 x_{i}(t), g_{2}\left(x_{i}(t)\right)=0.8 x_{i}(t)$, and the coupled matrix is chosen as

$$
\begin{aligned}
& A_{1}=\left(a_{i j}^{1}\right)=\left[\begin{array}{ccccc}
-1 & 1 & 0 & 0 & 0 \\
0 & -1 & 1 & 0 & 0 \\
0 & 0 & -1 & 1 & 0 \\
0 & 0 & 0 & -1 & 1 \\
1 & 0 & 0 & 0 & -1
\end{array}\right], \\
& A_{2}=\left(a_{i j}^{2}\right)=\left[\begin{array}{ccccc}
-1 & 0 & 1 & 0 & 0 \\
1 & -1 & 0 & 0 & 0 \\
0 & 1 & -2 & 1 & 0 \\
0 & 0 & 0 & -1 & 1 \\
0 & 1 & 0 & 0 & -1
\end{array}\right] .
\end{aligned}
$$

The synchronization state $s(t)$ satisfies

$$
\begin{aligned}
d s(t)= & {[C(r(t)) s(t)+B(r(t)) f(s(t))] d t } \\
& +g(t, s(t), r(t)) d \omega(t) .
\end{aligned}
$$

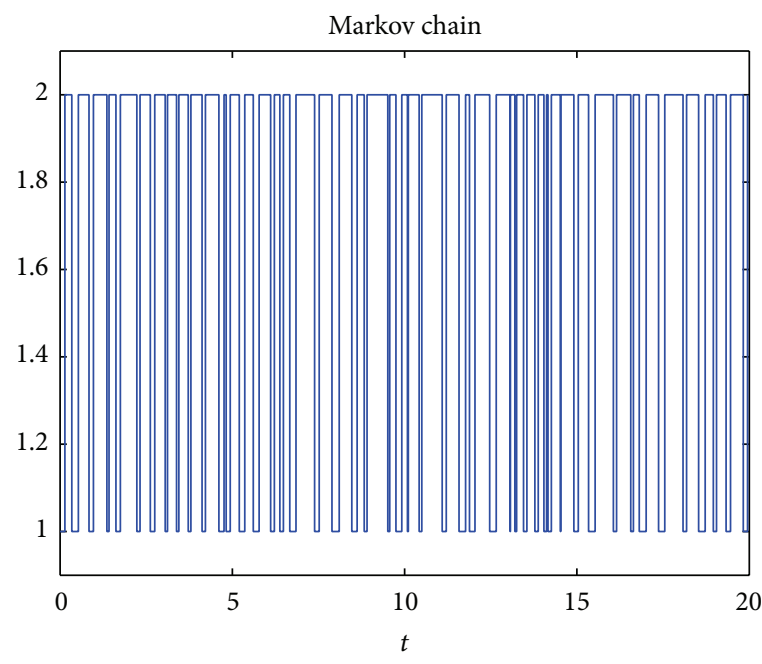

FIGURE 1: Markov chain generated by the probability transition matrix $\Pi_{1}$

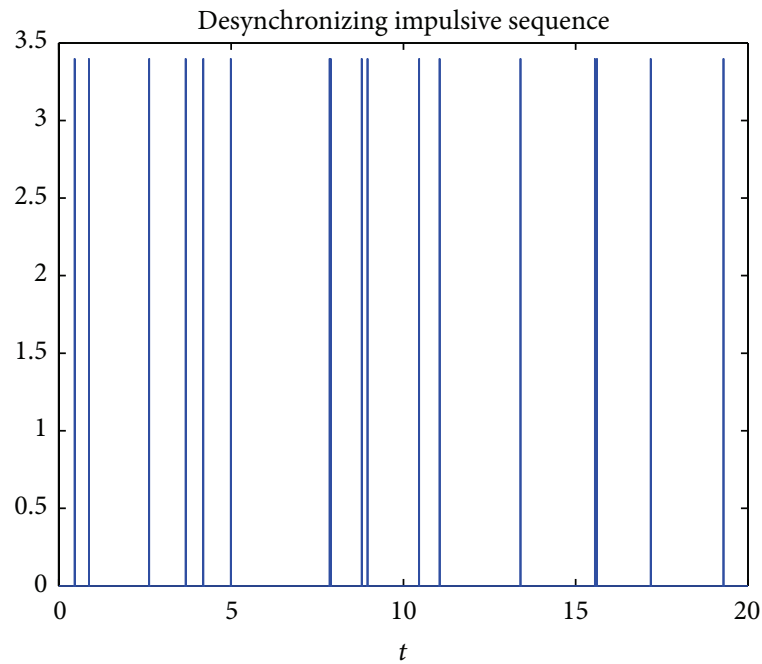

FIGURE 2: Desynchronizing impulsive sequence.

The nonlinear function $f(\cdot)$ satisfies the Lipschitz condition with $l=1$, so we get $\eta_{1}=-0.9147$ and $\eta_{2}=$ -0.7759 . Hence, $M=-\operatorname{diag}\left(\eta_{1}, \eta_{2}\right)-\Pi_{1}$ is a nonsingular $M$-matrix. Let $\vec{\alpha}=(0.2,0.2)^{T}$; we have $\left(q_{1}, q_{2}\right)^{T}=$ $M^{-1} \vec{\alpha}=[0.2481,0.2508]^{T}$. According to Theorem 9, if $T_{a}=4, N_{0}=12$, and $\varepsilon=0.05$, the stochastic complex network (33) is globally exponential synchronization in mean square when $\left|1+b_{i k}\right| \leq \sqrt{0.2481 / 0.2508} e^{(0.4 / 0.2508)-0.1}=$ 4.4347. For any $i$ and $k$, let $b_{i k}=3.4$; it means that all nodes have impulsive interference at the same time. The initial conditions for this simulation are $x\left(t_{0}\right)=$ $(1,2 ; 3,4 ; 5,6 ; 1.5,2.1 ; 3,2.4)$ and $s\left(t_{0}\right)=(8,9)$. The simulation results are given in Figures 1-4. Figure 1 shows a Markov chain generated by the probability transition matrix $\Pi_{1}$; Figure 2 shows the impulsive signal; the trajectories of the stochastic complex network (33) are shown in Figures 3 and 4 . It is clear that all nodes $x_{i}(t)$ tend to synchronization state $s(t)$. 
4.2. Example 2. Let $r(t)$ be a Markovian chain in the state space $S=\{1,2\}$ with the generator $\Pi_{2}=\left[\begin{array}{cc}-1 & 1 \\ 2 & -2\end{array}\right]$. Consider that a stochastic complex network consists of six nodes and two modes, each node in the network is a three-order Lorenz system if the Markov chain $r(t)=1$, while each node is a three-order Rössler system if $r(t)=2$. The stochastic complex network is described as follows:

$$
\begin{aligned}
d x_{i}(t)= & {\left[C(r(t)) x_{i}(t)+\bar{f}\left(x_{i}(t), r(t)\right)\right.} \\
& \left.+c(r(t)) \sum_{j=1}^{6} a_{i j}(r(t)) \Gamma(r(t)) x_{j}(t)\right] d t \\
& +g\left(t, x_{i}(t), r(t)\right) d \omega(t), \quad t \neq t_{k} \\
x_{i}\left(t_{k}\right)-x_{i}\left(t_{k}^{-}\right) & =b_{i k}\left(x_{i}(t)-s(t)\right), \quad t=t_{k}, \quad i=1,2, \ldots, 6,
\end{aligned}
$$

in which $x_{i}(t)=\left[x_{i 1}(t), x_{i 2}(t), x_{i 3}(t)\right]^{T}, \bar{f}_{1}\left(x_{i}(t)\right)=$ $0.1\left(0,-x_{i 1}(t) x_{i 3}(t), x_{i 1}(t) x_{i 2}(t)\right)^{T}, \bar{f}_{2}\left(x_{i}(t)\right)=0.5(0,0,0.2+$ $\left.x_{i 1}(t) x_{i 3}(t)\right)^{T}, c 1=c 2=1, \Gamma_{1}=0.5 I_{3}, \Gamma_{2}=0.8 I_{3}$, $C_{1}=0.1\left[\begin{array}{ccc}-10 & 10 & 0 \\ 28 & -1 & 0 \\ 0 & 0 & -8 / 3\end{array}\right], C_{2}=0.5\left[\begin{array}{ccc}0 & -1 & -1 \\ 1 & 0.2 & 0 \\ 0 & 0 & -5.7\end{array}\right], g_{1}\left(x_{i}(t)\right)=$ $0.5 x_{i}(t), g_{2}\left(x_{i}(t)\right)=0.2 x_{i}(t)$, and the coupled matrix is chosen as

$$
\begin{aligned}
A_{1}=\left(a_{i j}^{1}\right)= & {\left[\begin{array}{cccccc}
-5 & 1 & 1 & 1 & 1 & 1 \\
1 & -5 & 1 & 1 & 1 & 1 \\
1 & 1 & -5 & 1 & 1 & 1 \\
1 & 1 & 1 & -5 & 1 & 1 \\
1 & 1 & 1 & 1 & -5 & 1 \\
1 & 1 & 1 & 1 & 1 & -5
\end{array}\right], } \\
A_{2}=\left(a_{i j}^{2}\right)= & {\left[\begin{array}{cccccc}
-2 & 1 & 0 & 0 & 0 & 1 \\
1 & -2 & 1 & 0 & 0 & 0 \\
0 & 1 & -2 & 1 & 0 & 0 \\
0 & 0 & 1 & -2 & 1 & 0 \\
0 & 0 & 0 & 1 & -2 & 1 \\
1 & 0 & 0 & 0 & 1 & -2
\end{array}\right] . }
\end{aligned}
$$

The synchronization state $s(t)$ satisfies

$$
\begin{aligned}
d s(t)= & {[C(r(t)) s(t)+\bar{f}(s(t), r(t))] d t } \\
& +g(t, s(t), r(t)) d \omega(t) .
\end{aligned}
$$

The initial conditions for this simulation are $x\left(t_{0}\right)=$ $(-2,1,2 ; 3,-1,4 ; 3,-1,-2 ; 2.1,3,2.4 ;-1.2,2,2 ; 1,-1,-2)$ and $s\left(t_{0}\right)=(-3,-2,3)$. The nonlinear functions $\bar{f}_{1}(\cdot)$ and $\bar{f}_{2}(\cdot)$ satisfy the Lipschitz condition with $l_{1}=4.4733$ and $l_{2}=$ 6.0104 , so we get $\eta_{1}=12.0017$ and $\eta_{2}=12.2608$. Hence, $M=\operatorname{diag}(\eta, \eta)+\Pi_{2}$ is a nonsingular $M$-matrix. Let $\vec{\alpha}=$ $(2,2)^{T}$; we have $\left(q_{1}, q_{2}\right)^{T}=M^{-1} \vec{\alpha}=(0.1670,0.1624)^{T}$. If $T_{a}=0.1, N_{0}=2$, and $\varepsilon=0.1$, according to Theorem 13, the stochastic complex network (36) is globally exponential synchronization in mean square when $\mid 1+$ $b_{i k} \mid \leq \sqrt{0.1670 / 0.1624} e^{-(1 / 2)((2 / 0.1624)+0.1) \times 0.1}=0.5451$. In this

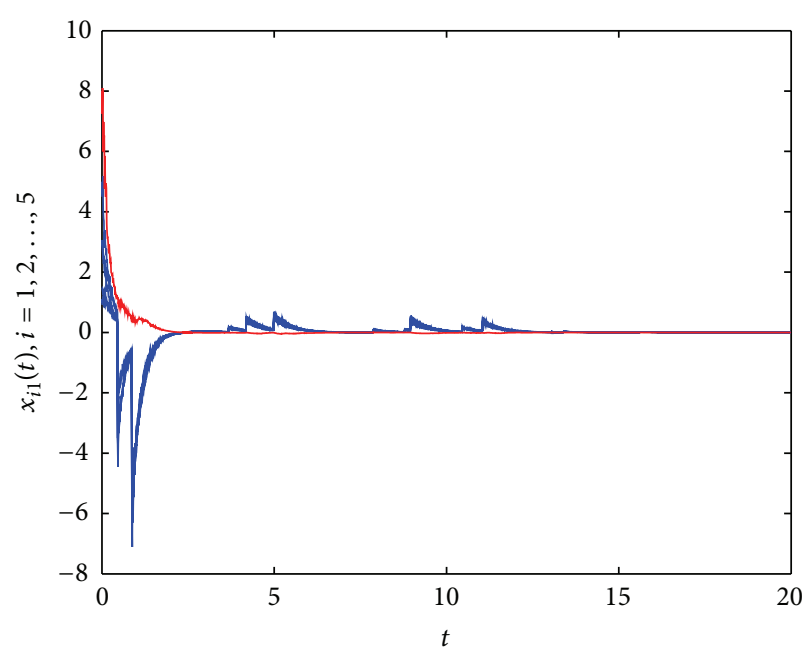

FIGURE 3: The trajectories of the state variables of $x_{i 1}(t)$ and $s_{1}(t)$ in stochastic complex network (33) with desynchronizing impulses.

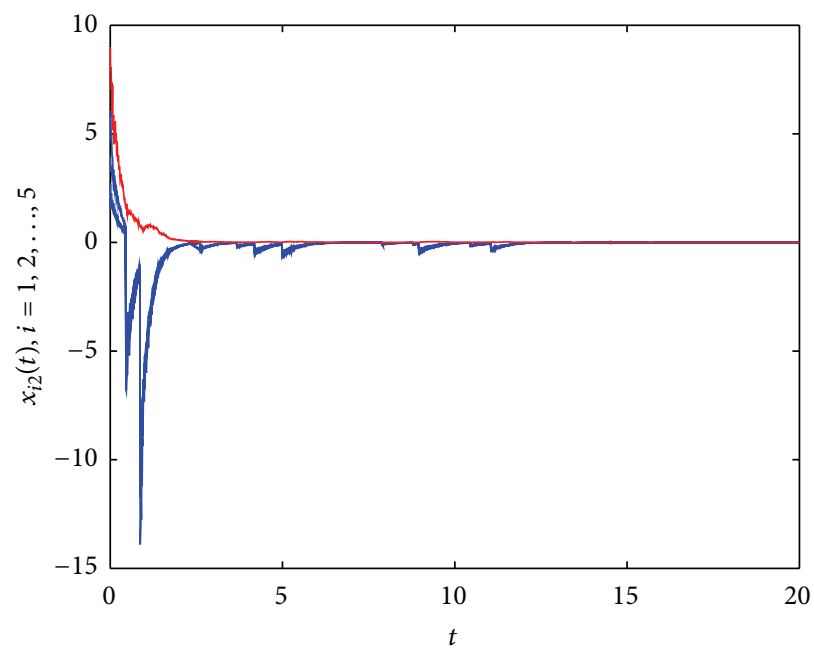

FIGURE 4: The trajectories of the state variables of $x_{i 2}(t)$ and $s_{2}(t)$ in stochastic complex network (33) with desynchronizing impulses.

simulation, let $b_{i k}=-0.5$. The simulation results are given in Figures 5-9. Figure 5 shows a Markov chain generated by the probability transition matrix $\Pi_{2}$; Figure 6 shows the impulsive signal. It can be seen clearly from Figures 7, 8, and 9 that all states of the stochastic complex network (36) tend to synchronization state $s(t)$.

\section{Conclusions}

In this paper, we have dealt with the exponential synchronization problem of complex dynamical networks with impulsive perturbations and Markovian switching. An $M$-matrix approach has been developed to solve the problem addressed. Some sufficient conditions are presented to guarantee the exponential synchronization of stochastic complex dynamical networks with impulsive perturbations and Markovian switching, which are independent of the upper bound of 


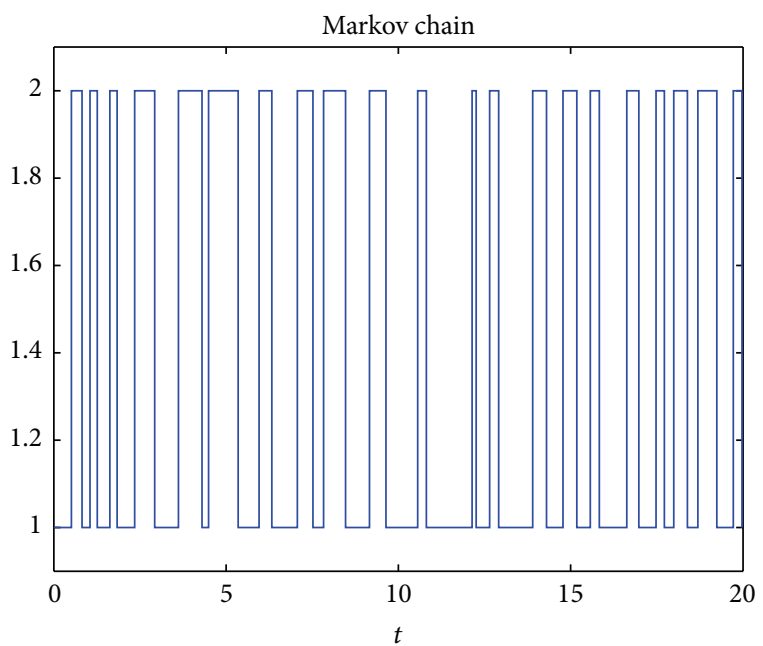

FIGURE 5: Markov chain generated by the probability transition matrix $\Pi_{2}$.

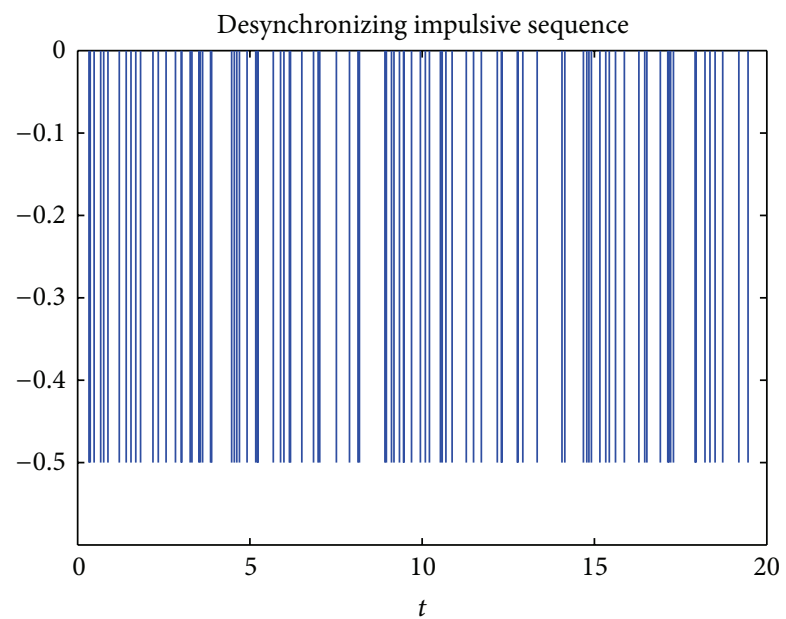

FIGURE 6: Synchronizing impulsive sequence.

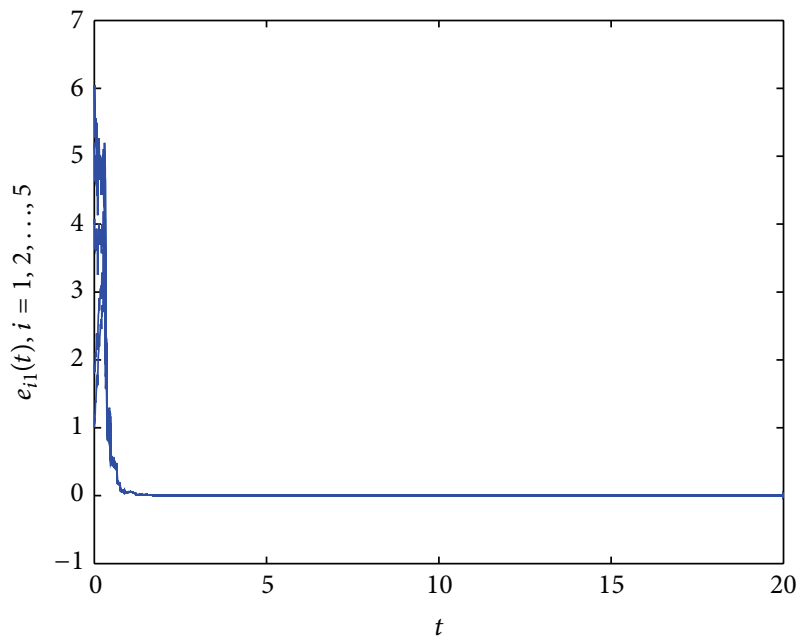

Figure 7: The trajectories of the error variables of $e_{i 1}(t)$ in stochastic complex network (36) with synchronizing impulses.

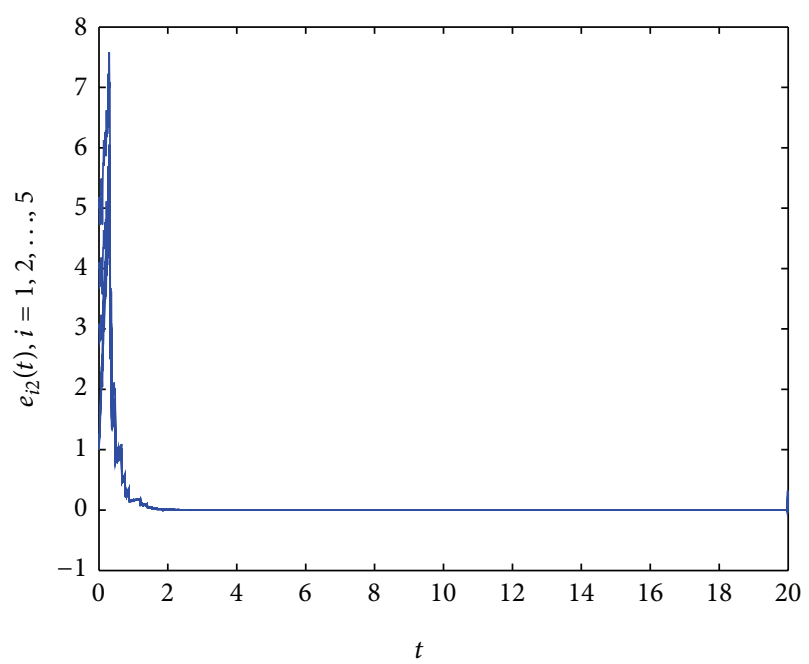

FIGURE 8: The trajectories of the error variables of $e_{i 2}(t)$ in stochastic complex network (36) with synchronizing impulses.

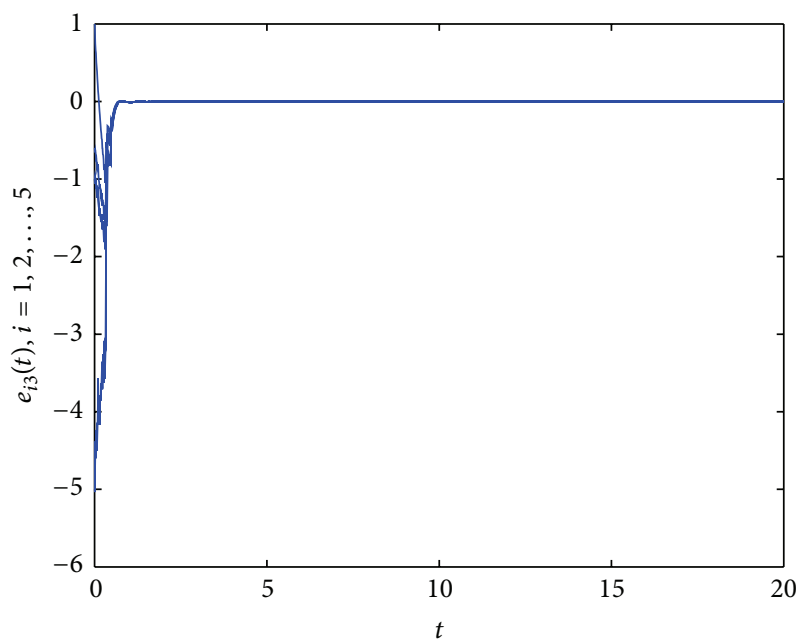

FIGURE 9: The trajectories of the error variables of $e_{i 3}(t)$ in stochastic complex network (36) with synchronizing impulses.

impulsive gain and Markovian switch. Finally, two numerical examples have been used to show the effectiveness of our results.

\section{Conflict of Interests}

The authors declare that there is no conflict of interests regarding the publication of this paper.

\section{Acknowledgments}

This work is supported by the National Natural Science Foundation of China (61075060), the Key Foundation Project of Shanghai (12JC1400400), and the Innovation Program of Shanghai Municipal Education Commission (12zz064, 13zz050). 


\section{References}

[1] S. H. Strogatz, "Exploring complex networks," Nature, vol. 410, no. 6825 , pp. 268-276, 2001.

[2] S. Boccaletti, V. Latora, Y. Moreno, M. Chavez, and D.-U. Hwang, "Complex networks: structure and dynamics," Physics Reports, vol. 424, no. 4-5, pp. 175-308, 2006.

[3] A. Arenas, A. Díaz-Guilera, J. Kurths, Y. Moreno, and C. Zhou, "Synchronization in complex networks," Physics Reports, vol. 469, no. 3, pp. 93-153, 2008.

[4] Z. Li and G. Chen, "Global synchronization and asymptotic stability of complex dynamical networks," IEEE Transactions on Circuits and Systems II, vol. 53, no. 1, pp. 28-33, 2006.

[5] J. Wang, J. Feng, C. Xu, and Y. Zhao, "Cluster synchronization of nonlinearly-coupled complex networks with nonidentical nodes and asymmetrical coupling matrix," Nonlinear Dynamics, vol. 67, no. 2, pp. 1635-1646, 2012.

[6] X. Wang and G. Chen, "Synchronization in small-world dynamical networks," International Journal of Bifurcation and Chaos, vol. 12, pp. 2735-2749, 2012.

[7] Z.-G. Wu, J. H. Park, H. Su, B. Song, and J. Chu, "Exponential synchronization for complex dynamical networks with sampled-data," Journal of the Franklin Institute, vol. 349, pp. 841846, 2005.

[8] J. Lü, X. H. Yu, and G. R. Chen, "Chaos synchronization of general complex dynamical networks," Physica A, vol. 334, no. 1-2, pp. 281-302, 2004.

[9] Y. Tang and W. Wong, "Distributed synchronization of coupled neural networks via randomly occurring control," IEEE Transactions on Neural Networks and Learning Systems, vol. 24, pp. 435-447, 2013.

[10] Y. Tang, H. Gao, and W. Zou, "Distributed synchronization in networks of agent systems with nolinearities and random switchings," IEEE Transactions on Cybernetics, vol. 43, pp. 358370, 2013.

[11] B. Shen, Z. Wang, and X. Liu, "Sampled-data synchronization control of dynamical networks with stochastic sampling," IEEE Transactions on Automatic Control, vol. 57, no. 10, pp. 26442650, 2012.

[12] W. Zhou, T. Wang, J. Mou, and J. Fang, "Mean square exponential synchronization in Lagrange sense for uncertain complex dynamical networks," Journal of the Franklin Institute, vol. 349, no. 3, pp. 1267-1282, 2012.

[13] W. Zhou, D. Tong, Y. Gao, C. Ji, and H. Su, "Mode and delay-dependent adaptive exponential synchronization in $p$ th moment for stochastic delayed neural networks with Markovian switching," IEEE Transactions on Neural Networks and Learning Systems, vol. 4, pp. 662-668, 2012.

[14] H. Shen, S. Xu, J. Lu, and J. Zhou, "Passivity-based control for uncertain stochastic jumping systems with mode-dependent round-trip time delays," Journal of the Franklin Institute, vol. 349, no. 5, pp. 1665-1680, 2012.

[15] S. He and F. Liu, "Robust stabilization of stochastic Markovian jumping systems via proportional-integral control," Signal Processing, vol. 91, no. 11, pp. 2478-2486, 2011.

[16] D. Tong, Q. Zhu, W. Zhou, Y. Xu, and J. Fang, "Adaptive synchronization for stochastic T-S fuzzy neural networks with timedelay and Markovian jumping parameters," Neurocomputing, vol. 117, pp. 91-97, 2013.

[17] Y. Gao, W. Zhou, C. Ji, D. Tong, and J. Fang, "Globally exponential stability of stochastic neutral-type delayed neural networks with impulsive perturbations and Markovian switching," Nonlinear Dynamics, vol. 70, no. 3, pp. 2107-2116, 2012.

[18] M. Casey, "The dynamics of discrete-time computation, with application to recurrent neural networks and finite state machine extraction," Neural Computation, vol. 8, no. 6, pp. 1135$1178,1996$.

[19] Y. Liu, Z. Wang, and X. Liu, "Exponential synchronization of complex networks with Markovian jump and mixed delays," Physics Letters A, vol. 372, no. 22, pp. 3986-3998, 2008.

[20] W.-M. Chen, C.-S. Li, F.-Y. Chiang, and H.-C. Chao, "Jumping ant routing algorithm for sensor networks," Computer Communications, vol. 30, no. 14-15, pp. 2892-2903, 2007.

[21] Q. Zhu and J. Cao, "Exponential stability of stochastic neural networks with both Markovian jump parameters and mixed time delays," IEEE Transactions on Systems, Man, and Cybernetics B, vol. 41, no. 2, pp. 341-353, 2011.

[22] Z. Wu, P. Shi, H. Su, and J. Chu, "Stochastic synchronization of Markovian jump neural networks with time-varying delay using sampled-data," IEEE Transactions on Cybernetics, vol. 43, pp. 1796-1806, 2013.

[23] Z.-G. Wu, P. Shi, H. Su, and J. Chu, "Passivity analysis for discrete-time stochastic markovian jump neural networks with mixed time delays," IEEE Transactions on Neural Networks, vol. 22, no. 10, pp. 1566-1575, 2011.

[24] J. Lu, D. W. C. Ho, and J. Cao, "A unified synchronization criterion for impulsive dynamical networks," Automatica, vol. 46, no. 7, pp. 1215-1221, 2010.

[25] W. Zhang, Y. Tang, Q. Miao, and W. Du, "Exponential synchronization of coupled switched neural networks with modedependent impulsive effects," IEEE Transactions on Neural Networks and Learning Systems, vol. 24, pp. 1316-1326, 2013.

[26] S. Cai, J. Zhou, L. Xiang, and Z. Liu, "Robust impulsive synchronization of complex delayed dynamical networks," Physics Letters A, vol. 372, no. 30, pp. 4990-4995, 2008.

[27] Q. Wu, X. Lan, and J. Zhou, "Average consensus in delayed networks of dynamic agents with impulsive effects," Complex Sciences, vol. 4, pp. 1124-1138, 2009.

[28] P. Li, J. Cao, and Z. Wang, "Robust impulsive synchronization of coupled delayed neural networks with uncertainties," Physica A, vol. 373, pp. 261-272, 2007.

[29] J. Zhou, Q. J. Wu, and L. Xiang, "Pinning complex delayed dynamical networks by a single impulsive controller," IEEE Transactions on Circuits and Systems I, vol. 58, no. 12, pp. 28822893, 2011.

[30] J. Lu, J. Kurths, J. Cao, N. Mahdavi, and C. Huang, "Synchronization control for nonlinear stochastic dynamical networks: pinning impulsive strategy," IEEE Transactions on Neural Networks and Learning Systems, vol. 23, pp. 285-292, 2012.

[31] X. R. Mao and C. G. Yuan, Stochastic Differential Equations with Markovian Switching, Imperial College Press, London, UK, 2006. 


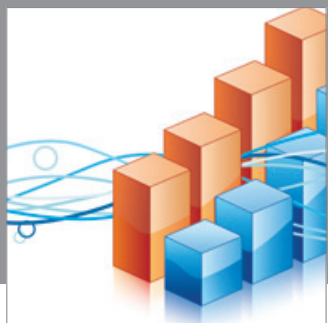

Advances in

Operations Research

mansans

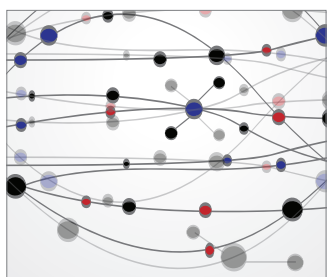

The Scientific World Journal
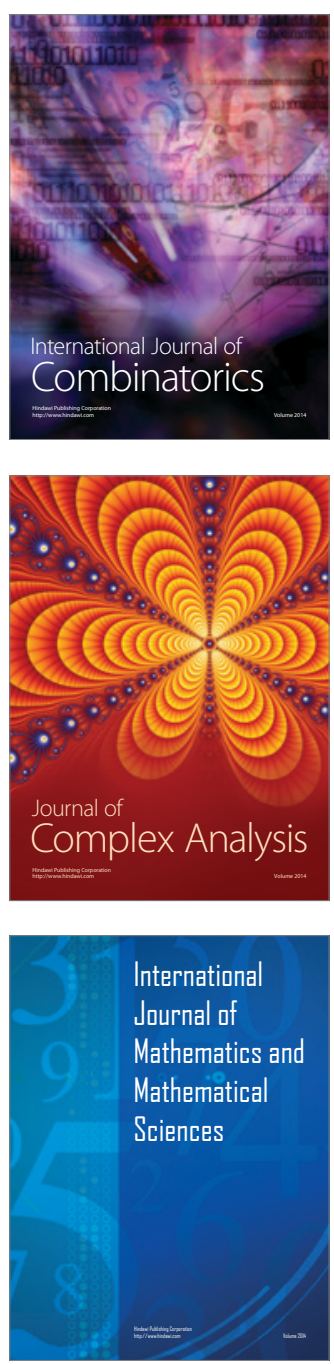
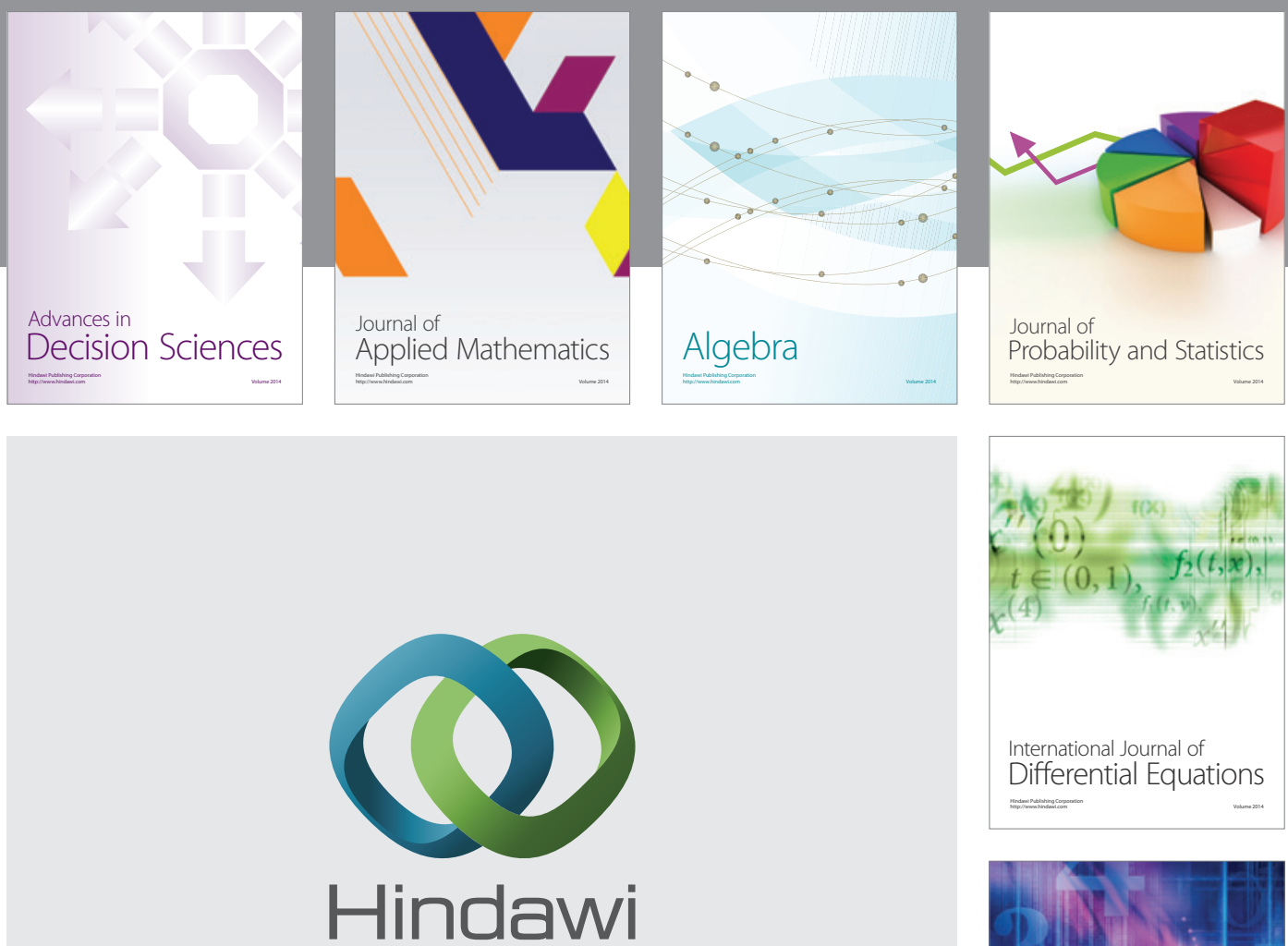

Submit your manuscripts at http://www.hindawi.com
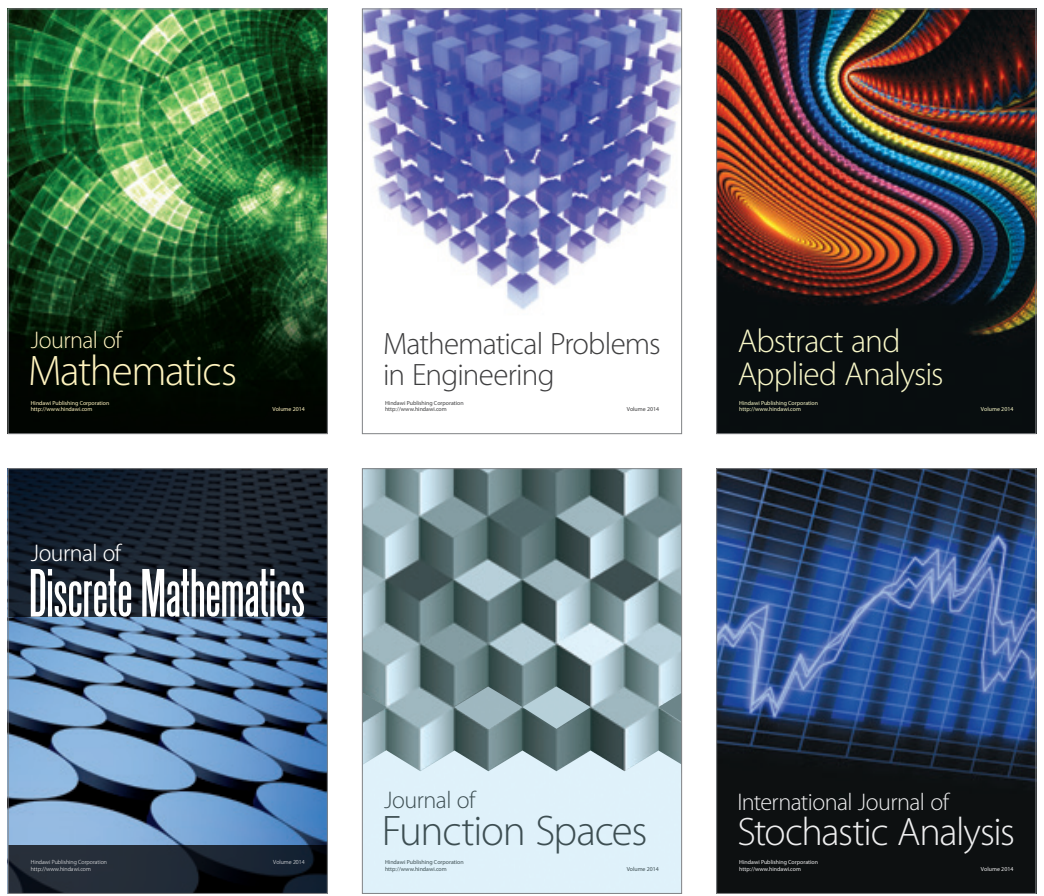

Journal of

Function Spaces

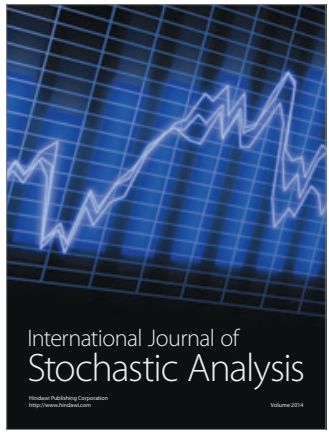

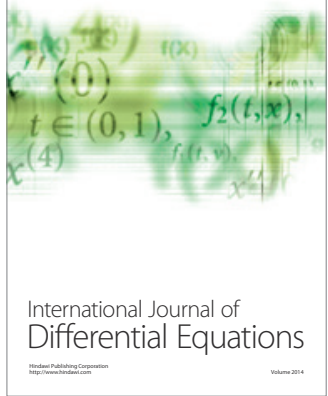
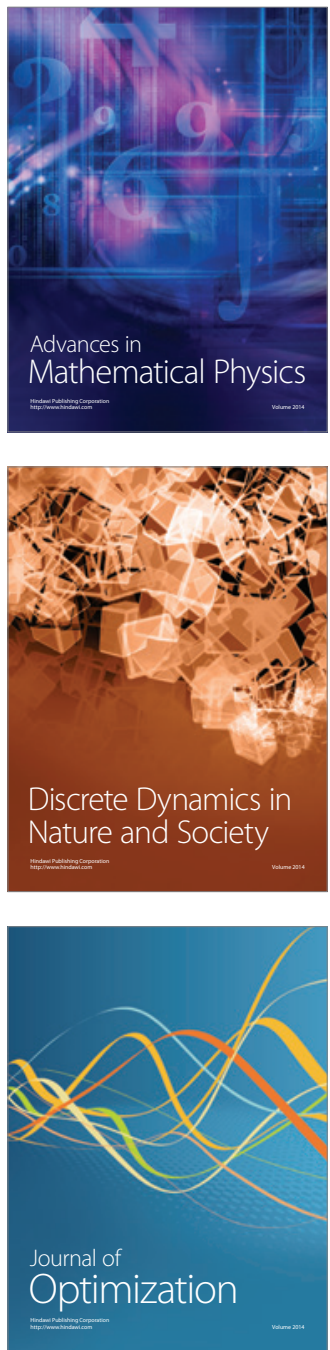